\title{
Linx
}

Revue des linguistes de l'université Paris X Nanterre

$68-69 \mid 2013$

Corpus et apprentissage du français

\section{Complémentarité des approches quantitatives et qualitatives dans l'étude du développement langagier}

L'exemple de l'acquisition des articles par le jeune enfant entre 1 et 3 ans

Tiphanie Bertin

\section{OpenEdition}

Journals

Édition électronique

URL : http://journals.openedition.org/linx/1483

DOI : $10.4000 / \operatorname{linx} .1483$

ISSN : 2118-9692

Éditeur

Presses universitaires de Paris Nanterre

Édition imprimée

Date de publication : 19 novembre 2013

Pagination : $33-48$

ISSN : 0246-8743

Référence électronique

Tiphanie Bertin, «Complémentarité des approches quantitatives et qualitatives dans l'étude du développement langagier », Linx [En ligne], 68-69 | 2013, mis en ligne le 19 novembre 2015, consulté le 10 décembre 2020. URL : http://journals.openedition.org/linx/1483; DOI : https://doi.org/10.4000/ $\operatorname{lin} x .1483$ 


\section{Complémentarité des approches quantitatives et qualitatives dans l'étude du développement langagier : l'exemple de l'acquisition des articles par le jeune enfant entre 1 et 3 ans}

Tiphanie Bertin, Université Sorbonne Nouvelle Paris 3, CLESTHLA

\section{Introduction}

Suite à l'important travail de recueil de données langagières enfantines de Brown (1973) ou encore de Bloom (1970,1973) dans les années 1970, nombre de chercheurs se sont inspirés de ces méthodes pour travailler sur des données longitudinales. Depuis, des bases de données se sont développées, qui regroupent des corpus plus ou moins longs, de données longitudinales ou transversales, de langues variées, d'enfants au développement typique ou atypique, ainsi que des outils pour analyser ces corpus. C'est le cas notamment de la base CHILDES (MacWhinney \& Snow, 1985), la plus développée à ce jour, mais également des bases françaises issues de projets comme TCOF (http://www.cnrtl.fr/corpus/tcof/) ou LEONARD (http://anr-leonard.ens-lyon.fr/). La problématique demeure toujours la même: un recueil de données et son traitement représente un temps de travail important qui rend difficile le travail sur un grand nombre d'enfants. Ainsi, ces bases de données offrent la possibilité de confronter et de compléter les résultats de ses propres données avec l'analyse de données déjà recueillies. Avec les outils proposés, il est possible à l'heure actuelle d'extraire des informations sur le langage de l'enfant de façon automatique et 
de les traiter statistiquement, comme le nombre de mots-types et de mots occurrences, le taux de diversité lexicale, ou encore la longueur moyenne d'énoncés, autant de mesures permettant de dégager des tendances pour décrire l'acquisition langagière et pour situer l'évolution des enfants. Ces approches statistiques sur un nombre important d'enfants renforcent la validité des résultats et mettent en avant une certaine significativité de ces derniers, permettant aux chercheurs de proposer des généralisations. Ainsi, des résultats importants sur le développement du langage sont basés sur ce type d'analyses de l'évolution langagière des enfants.

Mais qu'en est-il de ces méthodes quantitatives pour les études portant sur l'évolution langagière de l'enfant en lien avec les effets du langage que l'adulte lui adresse? Pour les recherches dont le but est de présenter comment l'enfant se rapproche progressivement du langage adulte et comment l'exposition à un tel langage l'y aide, il s'agit d'appliquer le même traitement statistique aux données de l'adulte. Les analyses consistent alors en recherches de corrélations entre certaines propriétés du langage de l'adulte et le développement de certains aspects langagiers chez l'enfant quelques mois plus tard (Furrow et al., 1979; Rondal, 1983). La question de ces méthodes quantitatives et statistiques se pose cependant pour les recherches portant sur les mécanismes interactionnels se retrouvant dans les dialogues entre adulte et enfant et contribuant aux processus d'acquisition du langage. Une approche quantitative statistique avec extraction automatique d'informations ne nous semble pas permettre d'illustrer complètement ces phénomènes.

À partir d'une étude sur les processus interactionnels d'appropriation des articles, nous proposons de montrer comment le fait de compléter cette approche quantitative par une analyse qualitative de séquences dialogiques du corpus permet de mieux illustrer le rôle de l'interaction adulte-enfant dans les processus d'appropriation du langage ${ }^{1}$.

\section{Problématique méthodologique : une majorité d'études centrées sur le langage de l'enfant}

Dans un cadre socio-interactionniste basé sur les théories de Vygotsky (1934/ 1991) et de Bruner (1983, 1987), le but général de notre étude est d’illustrer les mécanismes du processus interactionnel qui contribue à l'acquisition des articles et des clitiques sujets (avec une focalisation dans un premier temps sur la forme de ces éléments). Pour cela, nous avons travaillé sur des corpus longitudinaux de dialogues adulte-enfant, basés sur des livres illustrés, avec quatre enfants francophones âgés de 1 à 3 ans, enregistrés vingt minutes toutes les deux semaines pendant un an. Nous allons nous focaliser ici sur l'acquisition des articles chez un de ces enfants.

\footnotetext{
${ }^{1}$ Une illustration de la combinaison de ces deux types d'approches se retrouve également dans le numéro spécial du Journal of French Language Studies, «First Language Acquisition of French Grammar (from 10 months to 4 years old)» (Sekali, 2012) où les auteurs combinent analyses quantitatives et qualitatives dans leur étude de l'acquisition de différentes structures et marqueurs grammaticaux par plusieurs enfants, en lien avec l'input parental.
} 
Après avoir réalisé une analyse de l'évolution des productions de l'enfant, des formes précurseures des articles vers les formes standard, notre problématique méthodologique était la suivante : comment mettre en évidence les phénomènes interactionnels présents dans les dialogues entre adulte et enfant qui peuvent illustrer la façon dont ces échanges aident l'enfant à progresser dans son appropriation des articles?

Concernant les études déjà menées sur l'acquisition des morphèmes grammaticaux, la plupart sont centrées sur l'évolution du langage de l'enfant et portent sur le statut et l'évolution des formes précurseures de ces éléments, le plus souvent appelées «fillers» (Bassano, 2010a, 2010b ; Bolikova et al., 2009 ; Kilani-Schoch, 1998 ; Kilani-Schoch \& Dressler, 2000 ; Pepinsky et al., 2001 ; Peters, 2001). Ces études traitent de façon quantitative les différents aspects du langage de l'enfant, avec l'observation de l'évolution de la distribution des éléments dans le temps, de leur cotextes de production: syntaxiques, phonétiques, prosodiques. Autant d'approches qui traitent de l'évolution du langage de l'enfant mais pas de son lien possible avec le langage de l'adulte.

D'autres études, moins nombreuses, prennent en compte les productions de l'adulte dans l'acquisition de ces morphèmes grammaticaux. Pour beaucoup, il s'agit de mettre en parallèle la distribution des formes produites par l'enfant avec les formes produites par l'adulte afin de décrire la façon dont l'enfant progresse vers le modèle adulte et l'influence des caractéristiques de cet input sur cette évolution, comme par exemple la saillance phonétique de l'élément-cible, sa position dans l'énoncé ou sa fréquence dans l'input (Bassano, 2010b; Peters \& Menn, 1993; Veneziano, 1998, 2003 ; Veneziano \& Sinclair, 2000). L'objectif de ces études n'est alors pas d'illustrer un processus d'acquisition de ces éléments, ni même le rôle des dialogues de l'enfant avec l'adulte.

A l'heure actuelle, seulement quelques études s'intéressent aux conversations adulte-enfant dans l'acquisition des morphèmes grammaticaux. Certaines études s'appuient sur le langage de l'adulte pour déterminer la valeur discursive des premiers éléments grammaticaux produits par l'enfant dans son dialogue avec l'adulte (Allen, 2000 ; De Cat, 2004 ; Karmiloff-Smith, 1979 ; Salazar-Orvig et al., 2010a, 2010b; Serratrice, 2005). D'autres études, qui analysent le rôle de la conversation dans l'acquisition des éléments grammaticaux, portent principalement sur la morphologie verbale liée. À partir de séquences de tours de parole adjacents, il s'agit d'étudier les effets des productions de l'adulte dans ses dialogues avec l'enfant sur les productions de ce dernier, tant du point de vue des formes verbales utilisées (Veneziano \& Parisse, 2010), que des réactions aux productions morphologiques de l'enfant, erronées ou correctes (Kilani-Schoch et al., 2006, 2009). Bien qu'abordant la problématique du rôle de l'interaction adulte-enfant avec un objectif se rapprochant du nôtre, ces études portent sur la morphologie liée et ne traitent donc pas de la présence de fillers. Elles nous apportent cependant une approche méthodologique à adapter à notre objet d'étude.

L'objectif de notre étude s'inscrit alors dans un axe différent de la plupart des études sur l'acquisition des morphèmes grammaticaux libres : illustrer le fait que les 
Tiphanie Bertin

reprises et les reformulations de l'adulte en conversation avec un enfant constituent un moteur pour le processus d'appropriation des articles. Cela nécessite l'élaboration d'une méthodologie spécifique. Bien qu'ils n'aient pas été étudiés pour les éléments grammaticaux, ces mécanismes interactionnels ont été analysés dans le cadre de la mise en fonctionnement de la syntaxe chez les enfants, avec une approche descriptive du déroulement des échanges entre adulte et enfant (Canut, 2001; Canut et al., 2010 ; Canut \& Vertalier, 2010 ; Lentin, 1973, 1998/2009). Afin d'illustrer le rôle des interactions adulte-enfant dans le processus d'acquisition des articles, il nous a alors semblé important de ne pas nous limiter à un seul type d'analyse et de combiner approchse quantitative et qualitative des données d'interaction. Nous proposons dans cet article de décrire en quoi le recours à ces deux approches complémentaires nous semble indispensable pour rendre compte des processus interactionnels d'acquisition d'une langue maternelle, en présentant les apports et les limites de chacune dans le cadre de notre objet d'étude.

\section{Approche quantitative des données d'interaction : une analyse de l'évolution des reprises chez l'enfant et chez l'adulte}

Le but de notre analyse quantitative de l'évolution des reprises dans le dialogue est de faire ressortir l'importance des appuis de l'enfant sur les productions adultes pour produire des éléments en position d'articles, mais également d'observer les liens entre les verbalisations de ces deux locuteurs.

Au vu des propriétés discursives des articles, il semble peu pertinent d'étudier la reprise même de ces éléments. Ainsi, nous avons focalisé nos observations sur les reprises de groupes nominaux, et plus particulièrement celles contenant un nom identique, les plus repérées dans nos corpus. Dans le langage de l'enfant, trois configurations de groupes nominaux se repèrent :

- avec absence de forme $(\mathrm{O})$ : le nom n'est précédé d'aucun élément pouvant s'apparenter à un article ;

- avec forme de transition (FT) : le nom est précédé d'une forme précurseure d'un article ;

- avec forme finale (FF) : le nom est précédé d'une forme appropriée de l'article.

Nous avons différencié quatre contextes de production pour les groupes nominaux :

- en introduction : aucune mention n'a été faite précédemment par l'un ou l'autre des locuteurs ;

- en reprise immédiate: dans le tour de parole immédiatement adjacent à celui du l'interlocuteur ;

- en reprise différée : dans le tour de parole $n+1$ après celui de l'interlocuteur et dans les tours de parole suivants tant que le groupe nominal est repris ;

- en réintroduction : dans le tour de parole $n+2$ après celui de l'interlocuteur, s'il n'y a pas eu de reprise différée en $n+1$. 
Les deux questions auxquelles nous avons cherché à répondre avec cette analyse sont les suivantes : quelles sont les configurations des groupes nominaux produits par l'enfant dans les différents contextes de production, du plus proche au plus éloigné de la production adulte ? Et l'adulte reprend-il les groupes nominaux produits par l'enfant différemment selon leur configuration et dans quel type de reprise?

\subsection{Configuration des groupes nominaux produits par l'enfant dans les différents contextes de production}

Une de nos hypothèses de départ, basée sur les observations déjà réalisées pour le lexique ou la syntaxe, était que l'enfant produirait des formes appropriées d'articles d'abord en reprise immédiate puis progressivement dans des contextes de plus en plus éloignés des productions de l'adulte. Le Tableau 1 ci-dessous présente la distribution des différentes configurations de groupes nominaux pour chaque contexte de production (en fréquence d'apparition et en nombre d'occurrences) dans quatre corpus étudiés pour un même enfant $(\mathrm{C} 3, \mathrm{C} 7, \mathrm{C} 11, \mathrm{C} 16)$, pris à différents moments du processus d'appropriation des articles. Les fréquences d'apparition sont données par rapport à chaque contexte de production ce qui permet, pour chaque corpus, d'observer :

- le contexte de production dans lequel l'enfant produit plus souvent telle configuration;

- la configuration la plus produite dans chacun des contextes.

\begin{tabular}{|c|c|c|c|c|c|c|c|c|}
\hline \multirow{2}{*}{ Corpus } & \multicolumn{3}{|c|}{ GN introduits par l'enfant (E) } & \multirow{2}{*}{$\begin{array}{c}\text { Total de } \\
\text { GN } \\
\text { introduits } \\
\text { par E }\end{array}$} & \multicolumn{3}{|c|}{ GN réintroduits par $\mathbf{E}$} & \multirow{2}{*}{$\begin{array}{c}\text { Total de GN } \\
\text { réintroduits } \\
\text { par E } \\
\end{array}$} \\
\hline & $\mathbf{O}$ & FT & FF & & $\mathbf{O}$ & FT & FF & \\
\hline $\mathrm{C} 3$ & $\begin{array}{l}4 \\
23,53 \%\end{array}$ & $\begin{array}{l}9+ \\
1 \mathrm{FFFT} \\
58,82 \%\end{array}$ & $\begin{array}{l}3 \\
17,65 \%\end{array}$ & $\begin{array}{l}17 \\
100 \%\end{array}$ & $\begin{array}{l}17 \\
35,42 \%\end{array}$ & $\begin{array}{l}17 \\
35,42 \%\end{array}$ & $\begin{array}{l}14 \\
29,17 \%\end{array}$ & $\begin{array}{l}48 \\
100 \%\end{array}$ \\
\hline $\mathrm{C} 7$ & $\begin{array}{l}0 \\
0 \%\end{array}$ & $\begin{array}{l}12 \\
38,71 \%\end{array}$ & $\begin{array}{l}19 \\
61,29 \%\end{array}$ & $\begin{array}{l}31 \\
100 \%\end{array}$ & $\begin{array}{l}2 \\
7,14 \%\end{array}$ & $\begin{array}{l}2+1 \mathrm{FFFT} \\
10,71 \%\end{array}$ & $\begin{array}{l}23 \\
82,14 \%\end{array}$ & $\begin{array}{l}28 \\
100 \%\end{array}$ \\
\hline $\mathrm{C} 11$ & $\begin{array}{l}4 \\
16 \%\end{array}$ & $\begin{array}{l}3 \\
12 \%\end{array}$ & $\begin{array}{l}18 \\
72 \%\end{array}$ & $\begin{array}{l}25 \\
100 \%\end{array}$ & $\begin{array}{l}2 \\
9,52 \%\end{array}$ & $\begin{array}{l}0 \\
0 \%\end{array}$ & $\begin{array}{l}19 \\
90,48 \%\end{array}$ & $\begin{array}{l}21 \\
100 \%\end{array}$ \\
\hline C16 & $\begin{array}{l}2 \\
9,09 \%\end{array}$ & $\begin{array}{l}4 \\
18,18 \%\end{array}$ & $\begin{array}{l}16 \\
72,73 \%\end{array}$ & $\begin{array}{l}22 \\
100 \%\end{array}$ & $\begin{array}{l}1 \\
3,45 \%\end{array}$ & $\begin{array}{l}0 \\
0 \%\end{array}$ & $\begin{array}{l}28 \\
96,55 \%\end{array}$ & $\begin{array}{l}29 \\
100 \%\end{array}$ \\
\hline
\end{tabular}

\begin{tabular}{|c|c|c|c|c|c|c|c|c|}
\hline \multirow{2}{*}{ Corpus } & \multicolumn{3}{|c|}{ GN reprises différées par $\mathbf{E}$} & \multirow{2}{*}{$\begin{array}{l}\text { Total de } \\
\text { GN } \\
\text { reprises } \\
\text { différées } \\
\text { par } E\end{array}$} & \multicolumn{3}{|c|}{ GN reprises immédiates par $\mathbf{E}$} & \multirow{2}{*}{$\begin{array}{c}\text { Total de GN } \\
\text { reprises } \\
\text { immédiates } \\
\text { par E }\end{array}$} \\
\hline & $\mathbf{O}$ & FT & FF & & $\mathbf{O}$ & FT & FF & \\
\hline $\mathrm{C} 3$ & $\begin{array}{l}2 \\
10,53 \%\end{array}$ & $\begin{array}{l}11 \\
57,89 \%\end{array}$ & $\begin{array}{l}6 \\
31,58 \%\end{array}$ & $\begin{array}{l}19 \\
100 \%\end{array}$ & $\begin{array}{l}6 \\
16,22 \%\end{array}$ & $\begin{array}{l}15 \\
40,54 \%\end{array}$ & $\begin{array}{l}16 \\
43,24 \%\end{array}$ & $\begin{array}{l}37 \\
100 \%\end{array}$ \\
\hline $\mathrm{C} 7$ & $\begin{array}{l}0 \\
0 \% \\
\end{array}$ & $\begin{array}{l}1 \\
8,33 \% \\
\end{array}$ & $\begin{array}{l}11 \\
91,67 \% \\
\end{array}$ & $\begin{array}{l}12 \\
100 \% \\
\end{array}$ & $\begin{array}{l}6 \\
19,35 \% \\
\end{array}$ & $\begin{array}{l}7 \\
22,58 \% \\
\end{array}$ & $\begin{array}{l}18 \\
58,06 \% \\
\end{array}$ & $\begin{array}{l}31 \\
100 \% \\
\end{array}$ \\
\hline $\mathrm{C} 11$ & $\begin{array}{l}0 \\
0 \%\end{array}$ & $\begin{array}{l}0 \\
0 \%\end{array}$ & $\begin{array}{l}3 \\
100 \%\end{array}$ & $\begin{array}{l}3 \\
100 \%\end{array}$ & $\begin{array}{l}2 \\
20 \%\end{array}$ & $\begin{array}{l}2 \\
20 \%\end{array}$ & $\begin{array}{l}6 \\
60 \%\end{array}$ & $\begin{array}{l}10 \\
100 \%\end{array}$ \\
\hline $\mathrm{C} 16$ & $\begin{array}{l}0 \\
0 \% \\
\end{array}$ & $\begin{array}{l}0 \\
0 \% \\
\end{array}$ & $\begin{array}{l}6 \\
100 \% \\
\end{array}$ & $\begin{array}{l}6 \\
100 \% \\
\end{array}$ & $\begin{array}{l}1 \\
5,26 \% \\
\end{array}$ & $\begin{array}{ll}0 \\
0 \% \\
\end{array}$ & $\begin{array}{l}18 \\
94,74 \%\end{array}$ & $\begin{array}{l}19 \\
100 \% \\
\end{array}$ \\
\hline
\end{tabular}

Tableau 1 : Fréquence d'utilisation dans les corpus de Gaëtan des absences de formes, formes de transition et formes finales dans chaque contexte interactionnel de production des groupes nominaux 
Tiphanie Bertin

L'étude de ce tableau permet de constater que les groupes nominaux avec forme finale sont les plus produits d'abord en reprise immédiate (43,24\% de groupes nominaux avec forme finale en reprise immédiate en C3 contre 31,58\% en reprise différée, $29,17 \%$ en réintroduction et $17,65 \%$ en introduction). C'est également d'abord dans le contexte de reprise immédiate que les groupes nominaux avec formes finales sont produits majoritairement par rapport aux autres configurations $(43,24 \%$ de formes finales dans le corpus 3 contre $40,54 \%$ de formes de transition et 16,22\% d'absence de forme), alors que ce sont les formes de transition qui dominent dans les autres contextes dans le corpus 3, d'autant plus en introduction, le contexte le plus indépendant des productions de l'adulte. Cette première analyse des fréquences d'apparition semble donc valider notre hypothèse de départ : c'est dans le contexte le plus proche de l'adulte que l'enfant est d'abord capable de produire des formes appropriées d'articles. L'augmentation en C7, C11 et C16, de la part de groupes nominaux avec formes finales dans les trois contextes les plus éloignés de l'adulte va également dans ce sens avec par exemple une proportion de groupes nominaux avec forme finale en reprise différée qui passe de $31,58 \%$ en $\mathrm{C} 3$ à $91,67 \%$ en $\mathrm{C} 7$ puis à $100 \%$ en $\mathrm{C} 11$ et C16.

Cependant, selon notre hypothèse de départ, si l'enfant produit des formes appropriées d'articles d'abord à l'aide d'appuis immédiats sur les verbalisations de l'adulte puis progressivement dans des contextes plus éloignés, les groupes nominaux avec forme finale devraient se maintenir en majorité dans le contexte de reprise immédiate dans les corpus suivants et ceux avec absence de forme et forme de transition disparaitre d'abord dans ce contexte de production. Or, l'évolution présentée dans ce tableau fait ressortir que c'est dans ce contexte que ces deux configurations persistent le plus longtemps, avec en C7 une majorité d'absence de forme en reprise immédiate $(19,35 \%$ contre $7,14 \%$ en réintroduction et $0 \%$ en reprise différée et en introduction) et d'absence de forme et de formes de transition en C11 par rapport aux autres contextes. Cela met en évidence des appuis immédiats réguliers de l'enfant sur les verbalisations de l'adulte au cours de l'appropriation, avec production de groupes nominaux ne contenant pas une forme appropriée d'article.

L'utilisation d'une telle méthode quantitative pour le traitement de l'évolution des reprises de l'enfant nous permet donc de constater un rôle important de la reprise immédiate chez l'enfant au cours du processus d'appropriation des articles, qui serait le contexte privilégié de ses tâtonnements. Cependant, cela ne nous permet pas d'illustrer les mécanismes interactionnels du processus d'appropriation qui se jouent dans ce contexte, dans ces moments où les essais de l'enfant sur la production des articles se renforcent.

\subsection{Réactions de l'adulte aux différents groupes nominaux produits par l'enfant}

Le but de cette analyse quantitative des reprises de l'adulte est d'observer s'il répond différemment selon la configuration du groupe nominal produit par l'enfant (avec absence de forme, forme de transition, ou forme finale) et s'il s'ajuste donc aux 
Complémentarité des approches quantitatives et qualitatives dans l'étude du développement langagier...

productions de l'enfant notamment selon qu'elles sont conformes ou non à la langue standard. Nous avons pour cela calculé les taux de reprise et de non-reprise par l'adulte de chaque configuration de groupe nominal de l'enfant. Les résultats sont présentés dans le Tableau 2 ci-dessous. Puis, dans le cas d'une reprise, nous avons observé le type de reprise effectuée (immédiate, différée, réintroduction). Les résultats figurent dans le Tableau 3.

\begin{tabular}{|l|l|l|l|}
\hline \multirow{2}{*}{ Corpus } & \multicolumn{2}{|c|}{ GN avec Absence de forme } & \multirow{2}{*}{$\begin{array}{l}\text { Total de GN avec absence } \\
\text { de forme }\end{array}$} \\
\cline { 2 - 4 } & Non-repris & Repris & $28-100 \%$ \\
\hline C3 & $13-46,43 \%$ & $15-53,57 \%$ & $8-100 \%$ \\
\hline C7 & $1-12,50 \%$ & $7-87,50 \%$ & $8-100 \%$ \\
\hline C11 & $2-25 \%$ & $6-75 \%$ & $4-100 \%$ \\
\hline C16 & $1-25 \%$ & $3-75 \%$ & \\
\hline
\end{tabular}

\begin{tabular}{|l|l|l|l|}
\hline \multirow{2}{*}{ Corpus } & \multicolumn{2}{|l|}{ GN avec Forme de transition } & \multirow{2}{*}{$\begin{array}{l}\text { Total de GN avec forme } \\
\text { de transition }\end{array}$} \\
\cline { 2 - 4 } & Non-repris & Repris & $52-100 \%$ \\
\hline C3 & $26-50 \%$ & $26-50 \%$ & $23-100 \%$ \\
\hline C7 & $11-47,83 \%$ & $12-52,17 \%$ & $5-100 \%$ \\
\hline C11 & $0-0 \%$ & $5-100 \%$ & $4-100 \%$ \\
\hline C16 & $1-25 \%$ & $3-75 \%$ & \\
\hline
\end{tabular}

\begin{tabular}{|l|l|l|l|}
\hline \multirow{2}{*}{ Corpus } & \multicolumn{2}{|l|}{ GN avec Forme finale } & \multirow{2}{*}{$\begin{array}{l}\text { Total de GN avec forme } \\
\text { finale }\end{array}$} \\
\cline { 2 - 4 } & Non-repris & Repris & $39-100 \%$ \\
\hline C3 & $13-33,33 \%$ & $26-66,67 \%$ & $71-100 \%$ \\
\hline C7 & $30-42,25 \%$ & $41-57,75 \%$ & $46-100 \%$ \\
\hline C11 & $11-23,91 \%$ & $35-76,09 \%$ & $66-100 \%$ \\
\hline C16 & $14-21,21 \%$ & $52-78,79 \%$ & 6 \\
\hline
\end{tabular}

Tableau 2 : Fréquence de reprise et de non-reprise par l'adulte de chaque configuration de groupe nominal de Gaëtan

\begin{tabular}{|l|l|l|l|l|}
\hline \multirow{2}{*}{ Corpus } & \multicolumn{2}{|c|}{ GN avec Absence de forme repris } & \multirow{2}{*}{$\begin{array}{c}\text { Total de GN avec absence } \\
\text { de forme repris }\end{array}$} \\
\cline { 2 - 4 } & Reprise immédiate & Reprise Différée & Réintroduction & \\
\hline C3 & $13-86,67 \%$ & $1-6,67 \%$ & $1-6,67 \%$ & $15-100 \%$ \\
\hline C7 & $7-100 \%$ & $0-0 \%$ & $0-0 \%$ & $7-100 \%$ \\
\hline C11 & $6-100 \%$ & $0-0 \%$ & $0-0 \%$ & $6-100 \%$ \\
\hline C16 & $3-100 \%$ & $0-0 \%$ & $0-0 \%$ & $3-100 \%$ \\
\hline
\end{tabular}




\begin{tabular}{|l|l|l|l|l|}
\hline \multirow{2}{*}{ Corpus } & \multicolumn{3}{|c|}{ GN avec Forme finale repris } & \multirow{2}{*}{$\begin{array}{c}\text { Total de GN avec forme } \\
\text { finale repris }\end{array}$} \\
\cline { 2 - 4 } & Reprise immédiate & Reprise Différée & Réintroduction & \\
\hline C3 & $22-84,61 \%$ & $0-0 \%$ & $4-15,38 \%$ & $26-100 \%$ \\
\hline C7 & $37-90,24 \%$ & $1-2,44 \%$ & $3-7,32 \%$ & $41-100 \%$ \\
\hline C11 & $34-97,14 \%$ & $0-0 \%$ & $1-2,86 \%$ & $35-100 \%$ \\
\hline C16 & $48-92,31 \%$ & $3-5,77 \%$ & $1-1,92 \%$ & $52-100 \%$ \\
\hline
\end{tabular}

\begin{tabular}{|c|c|c|c|c|}
\hline \multirow{2}{*}{ Corpus } & \multicolumn{3}{|c|}{ GN avec Forme de transition repris } & \multirow{2}{*}{$\begin{array}{l}\text { Total de GN avec forme de } \\
\text { transition repris }\end{array}$} \\
\hline & Reprise immédiate & Reprise Différée & Réintroduction & \\
\hline $\mathrm{C} 3$ & $21-80,77 \%$ & $1-3,85 \%$ & $4-15,38 \%$ & $26-100 \%$ \\
\hline $\mathrm{C} 7$ & $11-91,67 \%$ & $0-0 \%$ & $1-8,33 \%$ & $12-100 \%$ \\
\hline C11 & $5-100 \%$ & $0-0 \%$ & $0-0 \%$ & $5-100 \%$ \\
\hline C16 & $3-100 \%$ & $0-0 \%$ & $0-0 \%$ & $3-100 \%$ \\
\hline
\end{tabular}

Tableau 3 :

Distribution des types de reprises de l'adulte pour chaque configuration de groupe nominal de Gaëtan

À partir de ces tableaux, nous pouvons observer que toutes les configurations de groupes nominaux produits par l'enfant sont plus souvent reprises par l'adulte que non reprises (Tableau 2). Nous pouvons également constater que, de façon générale, toutes les configurations sont de plus en plus souvent reprises, passant par exemple de $50 \%$ en C3 pour les formes de transition à $75 \%$ en C16. Ainsi, ces observations permettent de mettre en évidence que ce n'est pas parce que l'enfant avance dans l'appropriation des articles que l'adulte reprend moins ses productions. Le Tableau 3 permet en outre de constater que l'adulte reprend majoritairement en reprise immédiate toutes les configurations de groupes nominaux, et d'autant plus les groupes nominaux avec absence de forme ou forme de transition en fin d'appropriation, quand les groupes nominaux avec formes finales sont majoritaires dans le langage de l'enfant.

Ainsi, ces tableaux quantitatifs sur l'évolution des reprises chez l'adulte permettent de mettre en évidence l'évolution de la nature de ses reprises selon la configuration des groupes nominaux produits par l'enfant, à mesure que l'enfant progresse dans son appropriation des articles. L'adulte reprend de façon importante les verbalisations de l'enfant et l'interaction immédiate semble constituer un lieu privilégié de réponse à ses essais, d'autant plus utilisé que les formes en essai deviennent minoritaires.

\subsection{Apports et limites d'une analyse quantitative de l'évolution des reprises pour l'étude des processus interactionnels d'appropriation langagière}

Les résultats obtenus par cette analyse quantitative des reprises de l'enfant et de l'adulte suggèrent une adaptation du comportement interactionnel de l'adulte à l'évolution langagière de l'enfant. En outre, ces analyses mettent en évidence une importance de la 
Complémentarité des approches quantitatives et qualitatives dans l'étude du développement langagier...

reprise au cours de l'acquisition des articles et plus particulièrement de la reprise immédiate chez les deux locuteurs. Ces analyses nous orientent donc vers un type de séquences dialogiques qu'il serait pertinent d'observer pour compléter notre étude.

Si elle apporte un certain nombre de résultats, cette approche quantitative de l'analyse des interactions entre l'enfant et l'adulte ne propose qu'une analyse disjointe du langage de ces deux locuteurs. Il n'est par conséquent pas possible de saisir les effets plus fins des phénomènes interactionnels liés à l'utilisation de la reprise au cours du dialogue. Pour illustrer les processus interactionnels d'appropriation du langage, il nous semble nécessaire d'observer les effets mutuels de ces deux interlocuteurs sur leurs productions lors du déroulement de leurs échanges. Ainsi, après avoir étudié les modalités interactionnelles générales, il nous semble important de préciser les analyses en observant, à partir d'une analyse qualitative des conversations entre l'enfant et l'adulte, comment l'adulte réagit aux productions de l'enfant et quels en sont les effets sur les verbalisations de ce dernier.

\section{Analyse qualitative des échanges langagiers entre adulte et enfant}

\subsection{Rôle de la reprise immédiate dans le processus d'appropriation des articles}

L'objectif de cette analyse des dialogues entre adulte et enfant est de compléter la description du rôle du phénomène dialogique de la reprise dans l'acquisition des articles et de préciser l'importance de son fonctionnement dans le déroulement des processus d'appropriation langagière.

Nous allons pour cela observer deux séquences extraites des corpus 3 et 11 de Gaëtan (C3 et C11). Ces deux séquences illustrent les modifications dans le langage de l'enfant qui font suite à des reprises de l'adulte ${ }^{2}$ à deux moments différents de l'appropriation.

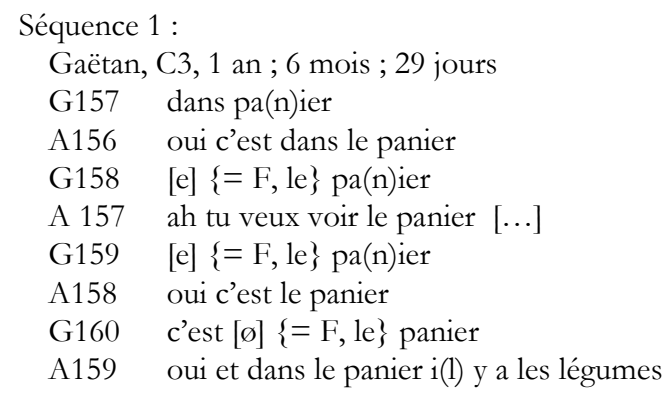

La séquence 1, qui se situe à une période où Gaëtan produit une majorité de formes de transition devant les noms, permet de constater comment les reprises de l'adulte du groupe nominal produit par l'enfant (A156, A157, A158) conduisent ce dernier à passer d'une absence de forme devant le nom (G157 dans panier) à la

\footnotetext{
${ }^{2}$ Les éléments transcrits sous leur forme phonétique suivis de $\{=\mathrm{F}$, article $\}$ sont des formes de transition, désignées par «F $\mathrm{F}$ et dont l'interprétation possible est donné par « article ».
} 
production d'une forme de transition (G158, G159 [e] panier) et à un changement de cette dernière (G160 c'est [0] panier). Il est également intéressant de noter ici que les reprises de l'adulte ne portent pas uniquement sur le groupe nominal mais l'intègrent dans une structure syntaxique plus large, qui entraine également l'enfant à allonger sa structure initiale, en reprenant celle proposée par l'adulte (G160).

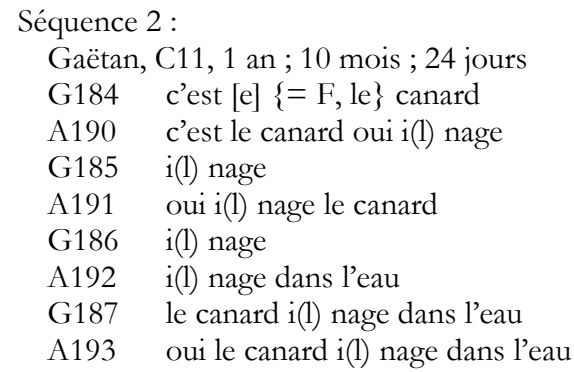

La séquence 2 se situe à une période où Gaëtan produit une majorité de formes finales devant les noms. Il est aisé de constater que l'adulte reprend toujours de façon presque systématique les productions de l'enfant, en les insérant dans des structures syntaxiques plus longues. À ce moment de l'appropriation, les reprises de l'adulte des groupes nominaux de l'enfant entrainent chez ce dernier la production d'une forme finale en lieu et place de la forme de transition utilisée initialement. Gaëtan commence ainsi par produire [e] canard (G184) et finit après plusieurs reprises de l'adulte par produire le canard (G187). D'autre part, l'allongement par l'adulte de la structure syntaxique utilisée par l'enfant entraine chez ce dernier un allongement de son énoncé de départ.

Ces deux séquences illustrent la façon dont les reprises de l'adulte amènent l'enfant à progresser sur la production des articles mais également sur les structures syntaxiques qui contiennent ces éléments, ce qui permet de resituer l'acquisition des articles dans la structuration progressive du langage.

\subsection{Influence d'une évolution simultanée sur plusieurs aspects linguistiques}

Le fait d'analyser l'évolution de la production des articles dans leur cotexte et contexte linguistiques permet de mieux appréhender certaines productions de l'enfant, où les essais sur les articles se trouvent influencés par des tâtonnements au niveau syntaxique ou encore lexical et inversement, comme dans les séquences 3 et 4 présentées ci-dessous.

Séquence 3 :

Sophie, C4, 2 ans ; 2 mois ; 22 jours

S106 c'est [valo] $\{=$ oiseau $\}$ là

A107 oui c'est un oiseau là

S107 $[\varnothing]\{=\mathrm{F}, /$ la, une $/\}$ vache

A108 hum

S108 là c'est vache

A109 oui là c'est la vache

S109 $[\varnothing]\{=\mathrm{F}, / \mathrm{un}, \mathrm{le} /\}$ oiseau 
La séquence 3 est extraite du corpus 4 de Sophie dans lequel elle produit une majorité de groupes nominaux avec absence de forme et avec forme de transition. Dans cet extrait, Sophie désigne à plusieurs reprises un élément présent sur l'illustration du livre qu'elle regarde avec l'adulte. La structure syntaxique qu'elle produit contient soit un élément présentatif " c'est» suivi du nom (S106 c'est [valo], S108 là c'est vache), soit une forme de transition suivie du nom (S10 [0] vache, S109 [0] oiseau), sans que ces deux éléments ne soient présents simultanément devant le nom. L'adulte reformule les verbalisations de l'enfant avec les structures syntaxiques adéquates. L'observation des productions de l'enfant dans cette séquence nous conduit à poser l'hypothèse d'une influence de la longueur de l'énoncé et donc des aspects syntaxiques sur l'acquisition de deux éléments de morphologie verbale libre, à savoir le présentatif et l'article.

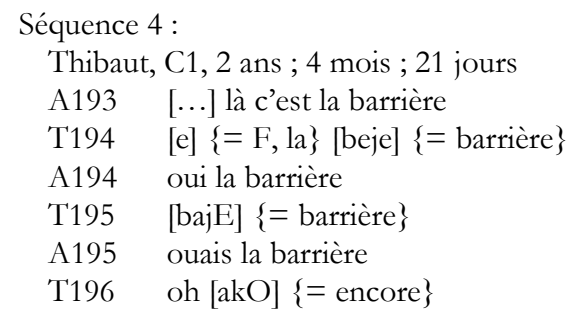

La séquence 4 est extraite du corpus 1 de Thibaut, dans lequel il produit une majorité de formes de transition devant les noms. L'adulte propose ici à l'enfant le groupe nominal la barrière (A193). L'enfant le reprend immédiatement avec une forme de transition [e] et le nom sous une forme non standard [beje] (T194). Puis suite à la reprise de l'adulte (A194), l'enfant produit de nouveau ce nom mais seul et sous une forme différente $[\mathrm{b} a j \mathrm{E}]$ qui n'est pas encore la forme conventionnelle, ce qui illustre un nouvel essai de production de ce mot par l'enfant. Cette production nous conduit à envisager une influence des tâtonnements lexicaux sur l'acquisition des articles et la production de formes de transition, ou finales.

D’autres séquences, que nous n'avons pas la place de présenter ici, mettent en évidence que ces deux influences, syntaxiques et lexicales, peuvent se retrouver simultanément dans un même énoncé.

\subsection{Apports et limites d'une analyse qualitative des dialogues adulte-enfant pour l'étude du processus interactionnel d'appropriation des articles}

En permettant l'analyse de la production d'éléments devant les noms dans leur contexte dialogique, l'étude qualitative de l'interaction permet d'illustrer de façon plus précise le rôle des reprises chez l'adulte et chez l'enfant dans le processus d'appropriation des articles, et de façon plus générale des différents aspects langagiers. L'analyse de ce fonctionnement interactionnel permet d'observer la façon dont les reprises de l'adulte, adaptées au niveau de développement langagier de l'enfant et à ses essais, aident ce dernier à progresser dans son appropriation mais également la façon dont les effets de ces reprises de l'adulte sur les productions de l'enfant évoluent à mesure qu'il progresse. Bien qu'elles ne soient pas systématiques, les reprises immédiates de l'enfant qui s'en suivent, lorsqu'elles contiennent des modifications, constituent des indices des effets 
de cette interaction et d'un processus en cours. Ce type d'observation qualitative permet ainsi d'illustrer le déroulement du processus d'appropriation des articles et de mettre en exergue le rôle d'une interaction immédiate et adaptée dans ce dernier.

En outre, en replaçant la production des articles dans leur cotexte linguistique, cette approche qualitative des corpus de dialogues adulte-enfant permet de prendre en compte les influences entre les tâtonnements de l'enfant au niveau des articles (absence de formes, formes de transition) et ses tâtonnements au niveau lexical ou syntaxique, avec des réponses de l'adulte à ces différents types de tâtonnements séparément ou simultanément, ce que ne permet pas l'analyse quantitative précédente qui traite les unités de façon isolée les unes des autres. Le processus d'appropriation des articles et des clitiques sujets se retrouve ainsi intégré dans le processus général d'appropriation du langage et donc dans l'acquisition simultanée des différents aspects langagiers.

\section{Conclusion}

L'ensemble de nos observations permet de mettre en évidence l'importance de combiner différentes approches pour étudier les processus d'appropriation du langage, et d'autant plus pour des approches interactionnelles.

Chaque approche prise séparément présente des avantages et des limites. Traitant séparément le langage de l'enfant et de l'adulte, une approche quantitative des corpus d'interaction ne permet pas de saisir le fonctionnement des processus interactionnels d'appropriation, mais elle permet de mettre en évidence des états de développement langagier et des modalités interactionnelles générales chez l'enfant et chez l'adulte. Elle peut servir alors de point de départ pour une analyse qualitative. Une approche qualitative des dialogues adulte-enfant permet quant à elle de saisir la façon dont les interactions adaptées de l'adulte, qui répond aux essais de l'enfant et lui propose des productions langagières allant légèrement au-delà de ce qu'il maitrise déjà, offrent à l'enfant des occasions de progresser dans son appropriation du langage. L'analyse de séquences dialogiques permet ainsi de saisir les mécanismes de la conversation moteurs des processus d'appropriation langagière, fondamentaux pour comprendre et expliquer la progression de l'enfant. Cependant, si elle n'est pas accompagnée d'une analyse quantitative préalable permettant de cibler les séquences à étudier pour l'objectif de l'étude, cette analyse de séquences dialogiques peut se révéler très couteuse en temps tant pour le repérage de séquences pertinentes dans les corpus que pour leur analyse.

Le croisement de ces deux analyses dans notre étude nous a permis dans un premier temps de faire ressortir l'importance de la reprise immédiate en corrélation avec l'évolution langagière de l'enfant et dans un second temps d'illustrer le rôle des reprises immédiates adaptées dans le processus d'appropriation des articles. 
Complémentarité des approches quantitatives et qualitatives dans l'étude du développement langagier...

Ainsi, le travail sur corpus et la complémentarité entre ces approches quantitatives et qualitatives nous semblent essentiels à exploiter lorsque l'on veut rendre compte de façon fine du rôle de l'interaction adulte-enfant dans les processus d'appropriation du langage, de la complexité du développement langagier et de son caractère multiaspectuel.

Cette analyse de séquences dialogiques mettant en évidence les modifications progressives dans les productions langagières de l'enfant qui s'approprie sa langue maternelle nous conduit à envisager un rapprochement avec les observations menées sur l'acquisition d'une langue seconde dans les conversations exolingues. Ainsi, Py (1989) et De Pietro et al. (1989) considèrent la conversation comme un constituant de l'acquisition d'une langue étrangère. Dans le contexte particulier de conversation asymétrique entre un locuteur natif et un locuteur apprenant, ces auteurs repèrent la présence de séquences dialogiques pouvant mener à l’acquisition de certains éléments de la langue-cible, notamment quand le locuteur natif répond aux difficultés langagières rencontrées par l'apprenant et que ce dernier s'en saisit pour poursuivre son énonciation. De telles séquences sont appelées « séquences potentiellement acquisitionnelles 》 (SPA), et sont relevées principalement en lien avec des difficultés lexicales, mais également syntaxiques (Jeanneret \& Py, 2002). Soulignant la complémentarité de ces deux types de SPA, Jeanneret et Py (2002) concluent à l'importance des mécanismes conversationnels dans l'acquisition d'une langue seconde. Il nous semble alors intéressant de rapprocher nos observations des séquences dialogiques entre adulte et enfant en cours d'acquisition de sa langue maternelle des SPA. L'asymétrie de compétences des locuteurs décrite pour la conversation exolingue se retrouve dans cette situation interactive dans laquelle nous avons noté l'importance, pour la progression de l'enfant, de l'interaction immédiate et adaptée de l'adulte. Cependant, la différence principale à ne pas omettre entre ces deux formats particuliers d'interaction se situe dans l'adaptation intuitive de l'adulte aux productions de l'enfant, différente de celle du locuteur natif qui répond à un « apprenant » cherchant consciemment à progresser.

Le travail sur corpus de dialogues adulte-enfant et la complémentarité dans les méthodes d'analyse posent de nouveau la question de la représentativité des données, puisqu'un traitement qualitatif des dialogues entre adulte et enfant requiert un temps certain. La mutualisation des données, des méthodes et des résultats, pour un travail collaboratif sur une problématique commune, à partir d'un vaste ensemble de corpus prend alors tout son sens. D'autant plus quand les résultats des recherches sur l'acquisition du français langue seconde, et d'une langue seconde en général, peuvent avoir un apport pour les recherches en français langue maternelle, et que l'objectif est bien de décrire les phénomènes interactionnels en lien avec les processus d'appropriation langagière. 


\section{Bibliographie}

ALLEN, S. (2000), "A discourse-pragmatic explanation for argument representation in child Inuktitut », Linguistics, 38/3, p. 483-521.

BASSANO, D. (2010a), « L'acquisition des verbes en français : un exemple de l'interface lexique / grammaire », Synergie France, 6, p. 27-39.

BASSANO, D. (2010b), «L'acquisition du déterminant nominal en français : une construction progressive et interactive de la grammaire», Cognitextes, 5, http://cognitextes.revues. $\operatorname{org} / 315$.

BLOOM, L. (1970), Language Development: Form and Function in Emerging Grammars, Cambridge MA, MIT Press.

Bloom, L. (1973), One Word at a Time. The Use of Single Word Utterances Before Syntax, La Haye, Mouton.

Bolikova, A., Kupish, T., Ozcelik, O., SAdlier-Brown, E. (2009), «Fillers as functional categories : evidence from German-English bilingual acquisition », dans, J. Crawford et al. (éds), Proceedings of the third conference on Generative Approaches to Language Acquisition North America (GALANA), Somerville, Cascadilla Proceedings Project, p. 1-12.

Brown, R. (1973), A First Language: The Early Stages, Cambridge MA, Harvard University Press.

BRUNER, J.S. (1983), Le développement de l'enfant : savoir-faire, savoir-dire, Paris, Presses Universitaires de France.

BRUNER, J.S. (1987), Comment les enfants apprennent à parler, Paris, Retz.

CANUT, E. (2001), Évolution de la syntaxe et de l'ancrage énonciatif dans des narrations d'enfants de moins de six ans. Interaction langagière entre adulte, enfant et livre, Villeneuve d'Ascq, Presses Universitaires du Septentrion.

CANUt, E., BoCÉRÉAN, C. \& ANDrÉ, V. (2010), «De l'apprentissage au développement : une approche interactionniste de l'acquisition des constructions syntaxiques complexes chez l'enfant de 3 à 6 ans ", dans F. Neveu. et al. (éds), Congrès Mondial de Linguistique Française CMLF 2010, Paris, Institut de Linguistique française, p. 1471-1487.

CAnut, E. \& Vertalier, M. (2010), «Étudier la complexité syntaxique chez l'enfant de moins de 6 ans dans une perspective interactionnelle : choix d'une méthodologie qualitative ", dans J. Bernicot, E. Veneziano, M. Musiol \& A. Bert-Erboul (éds), Interactions verbales et acquisition du langage, Paris, L'Harmattan, p. 239-260.

DE CAT, C. (2004), «Early pragmatic competence and its implication regarding the null subject phenomenon », dans R. Bok-Bennema et al. (éds), Romance Languages and Linguistic Theory 2002, Amsterdam, John Benjamins, p. 17-32.

De Pietro, J.F., Matthey, M. \& Py, B., (1989), «Acquisition et contrat didactique : les séquences potentiellement acquisitionnelles dans la conversation exolingue », dans L. Gajo, M. Matthey, D. Moore \& C. Serra (éds), 2004, Un parcours au contact des langues. Textes de Bernard Py commentés, Paris, Didier, p. 79-124. 
Complémentarité des approches quantitatives et qualitatives dans l'étude du développement langagier...

Furrow, D., Nelson, K. \& Benedict, H. (1979), « Mothers’ speech to children and syntactic development : some simple relationship ", Journal of Child Language, 6/3, p. 423-442.

JEANNERET, T. \& PY, B. (2002), «Traitement interactif de structures syntaxiques dans une perspective acquisitionnelle », dans F. Cicurel \& D. Véronique (éds), Discours, action et appropriation des langues, Paris, Presses de la Sorbonne Nouvelle, p. 37-52.

KARMiloff-Smith, A. (1979), A Functionnal Approach to Child Language: A Study of Determiners and Reference, Cambridge, Cambrige University Press.

KILANI-SCHOCH, M. (1998), «Construction des morphèmes grammaticaux dans les phases préet proto-morphologiques d'un corpus d'acquisition du français ", Verbum, 20/1, p. 73-86.

Kilani-Schoch, M., Balciuniene, I, Korecky-Kröll, K., LaAha, S. \& Dressler, W. U. (2009), « On the role of pragmatics in child directed speech for the acquisition of verb morphology », Journal of Pragmatics, 41/2, p. 219-239.

KILANI-SCHOCH, M., Dressler, W., LAAHA, S. \& K. KoreCKY-KRÖll (2006), «Réactions adultes aux productions morphologiques des enfants ", La linguistique, 42/2, p. 51-66.

KILANI-SCHOCH, M. \& DressLER, W. U. (2000), "Are fillers as precursors of morphemes relevant for morphological theory? A case study from the acquisition of French », dans W. U. Dressler, O. E. Pfeiffer, M. Pöchtrager \& J. R. Revison (éds), Morphological Analysis in Comparison, Amsterdam, John Benjamins, p. 89-111.

LENTIN, L. (1973), «Interactions adulte-enfant au cours de l'acquisition du langage », Études de linguistique appliquée, 9, p. 9-50.

LENTIN, L. (2009) [réed. 1998], Apprendre à penser, parler, lire, écrire : acquisition du langage oral et écrit, Paris, ESF.

Macwhinney, B. \& C. SNOW (1985), «The Child Language Data Exchange System », Journal of Child Language, 12, p. 271-296.

Pepinsky, T., Demuth, K. \& RoArk, B. (2001), «The status of 'filler syllables' in children's early speech », dans A. Do, L. Dominguez \& A. Johansen (éds), Proceedings of BUCLD 25, Somerville, Cascadilla Press, p. 575-586.

Peters, A. M. (2001), «Filler syllables: what is their status in emerging grammars? ", Journal of Child Language, 28/1, p.229-242.

Peters, A. M. \& MENN, L. (1993), «False starts and filler syllables: ways to learn grammatical morphemes », Language, 69/4, p. 742-777.

PY, B. (1989), «L'acquisition vue dans la perspective de l'interaction », DRLAV, 41, p.83-100.

Rondal, J. A. (1983), L'interaction adulte-enfant et la construction du langage, Bruxelles, Mardaga.

Salazar-Orvig, A., Marcos, H., Morgenstern, A., Hassan, R., Leber-Marin, J. \& PARES, J. (2010a), "Dialogical factors in toddlers' use of clitic pronouns », First Language, 30/3-4, p. 375-402.

Salazar-Orvig A., Marcos H., Morgenstern A., Hassan R., Leber-Marin J. \& Pares J. (2010b), «Dialogical beginnings of anaphora: the use of third person pronouns before the age of three », Journal of pragmatics, 42/7, p. 1842-1865. 
Tiphanie Bertin

SEKALI, M., (éd.) (2012), «First language acquisition of French grammar (from 10 months to 4 years old) ", Journal of French Language Studies, 22/1.

SERRATRICE, L. (2005), "The role of discourse pragmatics in the acquisition of subjects in Italian », Applied Psycholinguistics, 26/3, p. 437-462.

VenezianO, E. (1998), «Emergence de la morphologie grammaticale chez l'enfant, une continuité discontinue », Verbum, 20/1, p. 87-109.

VENEZIANO, E., (2003), "The emergence of noun and verb categories in the acquisition of French ", Psychology of Language and Communication, 7/1, p. 23-36.

Veneziano, E. \& PARISSE, C. (2010), «The acquisition of early verbs in French: assessing the role of conversation and of child-directed speech ", First Language, 30/3-4, p. 287-311.

Veneziano, E. \& Sinclair, H. (2000), «The changing status of 'filler syllables' on the way to grammatical morphemes ", Journal of Child Language, 27/3, p. 461-500.

Vygotsky, L. (1991) [trad. 1934], Pensée et langage, Paris, La Dispute. 